\title{
Index to Volume 7
}

(Numbers followed by asterisk refer to Society Abstracts)

Abaci, F. U. 161, 398*

Aballi, A. J. 353*, $357 *$

Abrahams, S. 300*

Abramowicz, M. 431*

Accto, T., Jr. 328*, 330*, 333*

$N$-Acetyl- $\beta$-glucosaminidase 751

Acid base homeostasis 126, 994

Acidosis 149

Acidosis, metabolic 712

Ackerman, B. D. 399*

Adam, P. A. J. 309*, 329*, 38I*

Adcbonojo, F. O. 353*

Adelman, R. D. 411 *

Adenine 729

Adenosine triphosphate 616

Adenylate cyclase 787

Adenyl cyclase 575, 878

Adinolfi, M. 317*

Adipose tissue 769

Adlard, B. P. F. 494, 883

Acrobic metabolism 794

Aftandelians, R. V. 373*

Agam, G. 66*

Agostino, R. 50 \%

Agrawal, H. C. 418*

Aharon, A. 370*

Ahmadian, Y. $415^{*}$

Ainbender, E. 224, 366*, 370*

Alaninc 184, 500, 983

Albala, M. 350*

Albuminuria 553

Alden, E. R. 302*

Aleck, K. 384*

Alexander, H. 293*

Alexander, H. 299*

Alford, C. A. 372*, 379*, 420*

Alkaline phosphatase 485

Allen, A. 329*

Alpert, E. 313*

Altenähr, E. 46 *

Altman, A. J. 351*

Altman, P. 429*

Altman, Y. 675

Altshulcr, G. P. 309*

Alvarado, J. 340*

Alvarez, M. N. $325^{*}$

Ament, M. E. 335*

Amino acid(s) 161, 184, 500, 818, 908, 994

Aminonucleoside nephrosis 553

Ammann, A. J. 364*, 369*
Amniocentesis 812, 863, 958

Amzel, V. 295*

Anagnostakis, D. 55*

Anast, C. $382 *, 386 *$

Ances, I. G. 509

Anderson, A. E. 322*

Anderson, J. M. 49*

Anderson, P. 369*, 378*

Andrcotti, G. 57*, 198

Andrews, B. F. 399*

Andria, G. 95

Anemia 937

Anencephaly 826

Angsusingha, K, 326*, 329*, 826

Ank, B. 380*

Ankeney, J. L. 302*

Anoxia 69i

Anserine 601

Anthony, B. F. 372*, 381*

Antiactivated factor $X 670$

Antibody response 103

Anticonvulsants 914

Antigen 103

Antithrombin III 670

Antley, R. M. 345*

Antonowicz, I. 335*, 337*

Antoon, A. 310*

Apnea 174, 569

Aradine, C. 729

Aranda, J. V. 184, 31 $\rceil^{*}, 382 *$, 393*, 399*

Arcilla, R. A. 299*

Arczynska, W. 50*, 126, 996

Arey, J. B. 304*

Argaman, M. 370*

Argininosuccinate synthetase 863

Argininosuccinic acid synthetase 700

Armendares, S. 371*

Arnaud, C. D. 485

Arnaud, S. B. 485

Arora, B. 336*

Arrobio, J. O. 373*

Arulanantham, K. 398*

Arvanitakis, S. N. 336*, 338*

Arylsulfatase A 751

Arzanian, M. 360*

Asakura, T. 360*

Aschinberg, L. C. 408*, 411*

Asphyxia 494

Aspnes, G. T. 343*, 358*, 359*

Assan, R. 53*
Asthma 627

Astor, S. H. 365*

Athanassiadis, S. 399*, 400*

Atherton, H. D. 404*, 405*, 408*

Atkinson, W. $408^{*}$

ATP 769

ATPase 494

Auerbach, V. H. 319*, 382*, 414*

Auld, P. A. M. 82, 396*, 403*, 569

Auricchio, S. 95

Autio, S. 52*

Averill, D. R. 37 $^{*}$

Avery, M. E. $425 *$, 842

Avioli, L. V. 333*, 392*

AvRuskin, T. W. $327 * 384 *$

Awa, S. 304*

Awdeh, B. 60*

Ayoub, E. M. 364*, 369*

Ayromlooi, J. 300*

Bach, F. H. 369*

Bacha, C. $349 *$

Bacon, G. E. 326*

Bacteriophage, modificd 675

Bader, P. I. 346*

Bachner, R. L. 351*

Baerlocher, K. 51*, 60*

Baez, S. 357*

Bailey, K. 398*

Bailic, M. D. 413*

Baird, H. W. 319*

Bakay, B. $342 *$

Bakcr, C. J. 289*

Baker, J. T. 412*

Baker, L. 294*, 385*

Baker, R. K. 385*

Balachandar, V. 316*, 336*, 385*, 394*

Baldwin, D. S. $416^{*}$

Balistreri, W. F. 319*

Ballard, C. A. $318^{*}$

Ballard, P. L. 308*

Ballard, R. A. 308*

Baluarte, J. 332*, 412*

Bancalari, E. H. 396*, 402*, 426*

Barak, Y. 353*, 361*

Bard, H. 310*

Barker, L. F. 370*

Barlow, M. J. 328*

Barltrop, D. 47*

Barnes, G. B. 294* 
Barnes, N. D. 326 *

Barnet, A. 420*

Barrett, C. T. 424*

Barrett, F. F. 289*, 373*

Barrett, J. 'T. 395*

Barta, R. A. 402*

Bartlett, G. S. 310*

Bartlett, K. G. 409*

Barton, L. 426*

Bashan, N. 66*

Bashore, R. A. 333*

Bass, H. N. 344*

Bassett, A. 303*

Bassi, J. A. 314*

Battaglia, F. C. 139, 309*, 314*, 396*, 794

Baublis, J. V. 378*

Bauditz, W. 45*

Bauer, C. $311^{*}$

Bauer, C. R. 395*

Bauer, R. 333*

Baum, F. K. 404*

Baumal, R. 367*

Baumann, J. B. 62*

Baumgartner, E. R. 58*

Baumgartnex, R. 64*

Bayard, F. 509

Bazaral, M. 368*

Beas, F. 326*

Beatty, C. H. 787

Bedell, J. H. 289*

Bédoucha, R. 64*

Behrman, R. E. 321*

Beitins, I. Z. 509

Bell, E. F. 588

Bellaire, J. 298*

Bellanti, J. A. 365*, 372*, 379*

Bellini, P. 198

Bclmonte, M. M. 326*, 410*

Belton, N. R. 49*

Benderli, A. 89

Benedict, W. F. 310*

Benest, D. $46^{*}$

Benke, P. J. 729

Bennett, B. 408*

Bennett, S. $315 *, 409 *$

Benton, J. W. 420*

Benz, H.-U. 53*

Benzing, G., III. 30I*

Beran, A. V. $427 *$

Berardinelli, J. L. 423*

Beratis, N. G. 224, 386*, 958

Bercu, B. 423*

Berg, W. v. 58*

Bergen, B. J. 978

Berger, R. 616

Bergseth, M. E. 395*

Bcrnard, B. 318*, 398*, 426*

Bernstein, C. W. 297*

Bcrnstein, J. M. 292*

Berry, A. M. 431*

Bessinger, F. B. 304*
Better, O. S. 89,712

Bhatia, M. 335*

Bhatnagar, R. M. 412*

Bianchi, R. F. 393*

Bidlingmaier, F. 901

Bieber, L. L. 309*, 310*

Bicdler, J. L. 388*

Bieri, J. G. 427*

Bierich, J. R. 62*

Bile acid 119

Biliary atresia, extrahepatic 119

Bingle, G. J. $346^{*}$

Bingol, N. 294*, 420*

Binkiewicz, A. 348*, 393*

Birth order 132

Birth weight 82, 132, 569

Black, I. F. S. $304 *, 414 *$

Blackburn, W. R. 308*, 310*

Blacklow, N. R. 292*

Blaese, R. M. 363*

Bläker, F. 61*

Blanc, W. A. $307^{*}, 321^{*}, 405^{*}$

Blanco, C. E. $306^{*}$

Blatman, S. 404*

Blau, E. 423*

Blaufox, M. D. $411 *, 413 *$

Bloch, K. J. 334*

Blood 509

Blood buffers 126

Blood, fetal 994

Bloom, A. D. $342 *, 700$

Bloomer, J. R. 334*

Blumberg, B. $365^{*}$

Boat, T. F. $340 *, 430 *, 607$

Bobik, C. M. $329 *$, 330*, 878

Bobik, J. R. 329*, 330*, 878

Bobo, R. 339*

Bocek, R. M. 787

Boda, D. 63*

Bode, H. H. 290*, 310*, 327*, 385*

Boedecker, H. 348*

Boerth, R. C. $318 *$

Boggs, D. 387*

Boggs, T. R. 403*

Bohne, M. 316*

Bohr effect 126, 994

Boineau, F. G. 412*

Bojanek, J. $50^{*}$

Boley, S. J. 336*

Bollinger, R. O. 353*

Bone morphology 757

Bonforte, R. J. 220, 366*, 369*, 958

Bongiovanni, A. M. 330*, 332*

Bonin, A. 335*

Boon, D. J. 335*

Borchert, P. 380*

Borella, L. 366*

Boroditsky, R. 306*

Bortolussi, R. 326*

Borut, D. 329*

Botstein, C. 358 *
Botstein, P. M. 290*

Bottini, E. 54*

Bottoggi, J. 397*

Boureau, M. 67 *

Bourland, B. J. 304*

Boxerbaum, B. $377^{*}$

Boyar, R. M. 323*, 328*

Boyd, G. 317*

Boyd, R. D. H. 794

Boyd, T. H. 357*

Bózkowa, K. 50* $65 *$

Braden, E. 416*

Bradford, W. 372*

Brady, J. P. 398*, 430*

Brahmacupta, N. 373*

Brain 527, 588, 691, 863

Brain, development 494

Braintwain, J. J. 376*

Branca, P. A. 291*, 401*

Branched chain amino acids 500

Branched chain amino aciduria 149

Brandt, C. D. 373*

Brandt, E. J. $366^{*}$

Brans, Y. 386*, 400*, 404*, 407*

Brasel, J. A. 307*

Braverman, S. 428*

Bray, G. A. 383*

Bray, P. F. 419*

Brechbühler, T. 58*, 64*

Breitenstein, M. 295*

Bremer, H. J. 53*, 64*, 66*

Brennan, J. F. 322*

Breslow, J. 39I*

Brill, A. B. 426* $431^{*}$

Bronchoconstriction 627

Brot, F. E. 384*

Brown, A. M. 292*, 324*, 719

Brown, D. M. 315*, 323*, 757

Brown, J. 569

Brown, J. W. $419^{*}$

Brown, R. S. 326*

Brunberg, J. A. 419*

Brunell, P. 373*

Bruns, G. 344*

Bryan, A. C. $291 *, 425^{*}, 429 *$

Bryan, H. 353*

Bryan, M. H. 291*

Brych, M. 326* 328*

Bucci, G. 50*

Buchert, E. 354*

Buckley, N. M. $303 *$

Buckley, R. 364*

Bucknall, W. E. 382*

Bucrgin-Wolff, A. 61*

Bühler, E. M. 56*

Bühler, U. 62*

Buist, N. R. M. 333*

Bull, D. L. 307*

Bunthrarungroj, T. 356*

Burghen, G. A. $325^{*}$

Burgio, G. R. 66* 
Burke, G. $375^{*}$

Burke, J. A. 335*

Burkhardt, H. 62*

Burks, C. M. 345 *

Burton, B. K. $419^{*}$

Buselmeier, T. J. 292*

Butanone 149

Butenandt, O. 901

Butler, D. G. 337*

Button, L. N. 301*

Buxton, S. 387*

Buzon, M. M. $411^{*}$

Byrd, N. 373*

Cabal, L. 306*

Cabalska, B. 65*

Cahill, L. T. 366*

Calcagno, P. L. 414*

Calcium 100, 485

Caleb, M. 354*

Calliumi, G. 50*

Camacho, A. M. 327*

Cameron, D. L. 371*

Camitta, B. 289*

Cammer, W. 422*

Camp, B. W. 294*

Campbell, A. G. M. 399*, 402*, 428*

Campbell, J. R. 333*

Campbcll, R. A. 333*, 391*, 412*, 426*

Cancilla, P. A. $389^{*}$

Canning, N. 347*

Capsular polysaccharide 103

Carbohydrate 787

Carbohydrate metabolism 494

Carcinogenesis 132

Cardos, S. F. 373*

Cardwell, S. E. $345^{*}$

Carey, W. B. 298*

Carl, R. 391*

Carmel, R. 341*

Carnosinase 601

Carnosine 601

Carr, M. C. $362 *$

Carsten, A. 336*

Carter, C. O. $56^{*}$

Carter, G. S. 298*

Carvajal, H. F. 410*

Carvajal, M. 297*

Cashore, W. J. 399*, 400*

Cassady, G. 386*, 400*, 404*, 407*

Cassidy, J. T. 363*

Cassin, S. 302*, 425*

Castell, R. 588

Castellanos, A. W. 302*

Castellanos, R. 306*

Castells, S. 327*, 385*, 415*

Catecholamines 983

Catz, C. S. $317 *$, 319*, 320*

Caul, J. 358*

C bands 39

Cederbaum, S. D. 423*
Cejka, J. 353*

Cereghino, J. J. 11 I

Cernichiari, E. $351^{*}$

Cha, C. $396^{*}$

Chabon, R. S. 294*

Chaimovitz, C. 89

Chakmakjian, Z. H. 71

Champagne, G. J. 406*

Chan, G. 423*, 430*

Chan, J. C. M. 315*, 412*, 712

Chan, N. 423*

Chance, G. W. 398*

Chandor, S. 349*

Chandra, R. D. 368*

Chandramouli, B. 386*

Chang, N. 341*

Channing, D. 334*

Chanock, R. M. 373*

Chantler, C. 414*

Chapman, S. S. 372*

Char, D. H. 292*

Charamclla, L. J. 379*

Charette, R. 376*

Charnock, E. L. 430*

Chaussain, J. L. 385*

Chavalitdhamrong, P. 384*, 388*

Chedru, M. F. 67*

Chemorcceptors 569

Chen, C. $415 *$

Chen, I. 323*

Chen, I. 408*

Chen, J.-H. 354*, 427*

Chen, S. Y. 316*, 336*, 385*, 394*

Cheong, I.-T. 410*, 415*

Chernack, W. J. 366*, 410*

Chernick, V. 20

Chesler, D. 296*

Ch'ien, L. T. 420 *

Childhood 901

Chin, F. T. 393*

Chiu, J. M. 412*

Chlebowski, R. 381*

Chloramphenicol 923

Cho, C. T. 373*, 400*

Cholestatic syndromes 119

Cholestyramine 119

Chondroitin sulfate 724,965

Chopra, D. R. 399*, 400*

Chorionic somatomammotropin 719

Chou, L. 346*

Chou, P. J. 399*

Chou, T.-C. 299*

Chow, A. W. 381*

Christiansen, R. O. 324*

Chromosome breaks 582

Chromosomes 39

Chuck, G. 389*

Chung, L. W. K. 318*

Chutorian, A. 422*

Chytil, F. 407*

Ciccimarra, F. 95
Cilia 607

Ciliary dyskinesia 220, 224, 958

Citrullinemia 700, 863

Clare, R. 54*

Clark, D. J. 373*

Clausen, J. 57*

Clawson, C. C. $350 *$

Clayton, C. W. 331*

Clements, R. S., Jr. 385*

Cleveland, W. W. 297*, 386*

C line 111

Clotting, disseminated intravascular 638

Cloutier, M. D. 325*

Coceani, F. 425 *

Cochran, W. 400*

Codish, S. D. $346^{*}$

Cohen, D. 400*

Cohen, F. 363*

Cohen, J. L. 945

Cohen, M. 299*, 365*, 380*

Cohen, M. E. 330*

Cohen, M. I. 319*, 334*, 336*

Cohen, M. L. 426*

Cohen, M. M. 696

Cohen, S. N. 401*

Cohn, J. D. 301*

Cold sensitivity 627

Cole, B. R. 409*

Cole, H. S. 385*

Cole, V. A. $50^{*}$

Colcman, M. 420*

Collagen 757

Colle, E. 184, 311*, 317*, 326*, 382*, 393*, $395^{*}$

Colletti, R. 299*

Collins, S. 313*

Collipp, P. J. 316*, 336*, 385*, 394*

Collu, R. 323*

Colón, A. R. 386*

Colonization, intestinal 659

Conca, L. 50*

Conde, C. $49 *$

Condron, C. J. 343*

Conerly, E. B. 645

Congenital infections 541

Conly, P. W. 297*

Connaughton, J. F. 319*

Connor, J. D. 373*

Conod, E. J. 220, 224, 958

Conover, J. H. 220, 224, 958

Constantopoulos, A. 366*

Convulsions 863

Cook, H. W. 394*

Cooper, M. D. 363*, 364*, 541

Coppa, G. 384*

Coproantibody 659

Coran, A. G. 306*

Corash, L. 360*, 798

Corbell, L. 59*

Cornblath, M. 309*

Corrigan, J. J., Jr. 354*, 374* 
Corsini, W. A. 413*

Costales, F. 353*

Costenbader, M. L. 380*

Corticosteroids 883

Cortisol 509

Cortisone 509

Counts, S. 413*

Cowger, M. L. 336*

Cox, B. J. 357*

Cox, F. E. 374*

Cracco, R. Q. 420*

Cramblett, H. G. 392*

Crandall, B. F. $344 * 346 *$

Crawford, J. D. 28, 290*, 310*, 327*, 385*

Critchlow, W. $415^{*}$

Croce, C. M. 342*

Crookston, M. C. 367*

Cropp, G. J. A. $427 *$

Crosby, J. M. 426*

Crumrinc, R. 396*

Cruskin, A. 332*

Cuendet, G. S. 383*, 983

Cuisinicr-Gleizes, P. 46*

Cukier, J. O. 416*

Cunningham, M. D. 354*, 406*

Curtius, H.-C. $51 *$

Cystathionase 527, 908

Cystathionine synthase 645

Cyst(c)ine 527, 908

Cystic fibrosis of the pancreas 220, 224, 958

Cytochalasin B 745

Cytochrome c 923

Cytogenetics 39

Cytryn, L. 420*

D'Ablaing, G., III 426*

Dacou-Voutetakis, C. 55 *

Dahlquist, G. 49*

Daily, W. J. R. 398*, 404*, 431*

Dakroury, A. 415*

Dallman, P. R. $307 *$, 315*

Dancis, J. 192

Danes, B. S. 724

D'Angio, G. 361*

Danicl, S. S. $397^{*}$

Danilowicz, D. 302*

Danis, R. K. 330 **

Danon, M. 327*

Daum, F. 334*, 336*

Daum, R. S. 149

Davachi, F. 301*

Davenport, S. L. H. 410*

David, I. 382*, 386*

David, R. B. 354*

Davies, D. P. $55^{*}$

Davis, C. W. C. $379 *, 541$

Davis, R. H. 427 *

Davis, S. D. $374^{*}$

Davis, S. E. $341^{*}$

Davison, A. N. 48*

Dawber, N. H. $334^{*}, 401^{*}$
Dawson, G. 684

Day, G. M. 398*

Dayton, P. G. 321*

dcBelle, R. 292*

Debove, F. $46^{*}$

DeChatelet, L. R. 364*

Deckelbaum, R. J. $340 *$

Deckert, F. W. 317*, 322*

De Coello, S. 312*

Deely, W. J. 30I*

Deforest, A. 375*

dcGuzman, A. L. 302*

Delamater, P. V. 388*, 407*

del annoy, C. W. 411*

De la Rosa, L. 37 l* $^{*}$

deLemos, R. A. 396*, 425*

de Leuchtenberg, N. 425*

deLevie, M. 328*

Del Guercio, L. R. M. 301*

Delivoria-Papadopoulos, M. 291*, 401*, $427 *$

Delorme, A. 46*

DeLuca, H. 387*

Delvin, D. 149

Denham, B. 290*

Den Tandt, W. R. 346*

Dentinger, M. P. 419*

2-Deoxy-D-glucose 983

Depp, R. 329*

Deren, J. J. $336^{*}$

Der Kaloustian, V. M. 60*

Dermatoglyphics $111,215,620$

Dery, P. 408*

Desai, J. N. 354*

Desautel, M. 324*

De Souza, S. W. 494

Developmental biochemistry $1,75,95,575$, $696,724,908$

Devclopmental physiology 527, 901, 931

DeVivo, D. 500

Diabetes mellitus 184

Diarrhea 161

Diamond, I. 352*, 418*, 420*

Diamond, L. K. 858

Diamond, R. 394*

Dicker, L. 354*

Dickerman, J. D. $355 *$

Dickerson, J. W. T. 778

Diebler, M. F. 385*

DiFcrrante, N. $384^{*}$

DiGeorge, A. M. 363*, 368*, 382*

DiLiberti, J. H. 382*

DiMauro, S. 739, 745

Dimich, I. 299*

Diserens, H. W. 396*

Distler, J. J. 393*

Djerassi, I. 352*

DNA 696

Dobbing, J. 494

Docrshuk, C. F. 430*

Doherty, R. A. $319 *, 351$ *
Dolanski, E. $405^{*}, 407^{*}$

Dollery, C. T. 54*

Dolney, A. M. 426*

Donaldson, R. M. 292*

Dong, L. 403*

Donncli, G. N. 59*, 391*

Dooley, R. 365*

L-Dopa $7 \mathrm{I}$

Dorfman, A. 372*, 384*

Dorst, J. 348*

Doty, D. B. 419*

Doty, S. B. 13

Douglas, C. 724

Downing, S. E. 301*

Downing, S. J. 316*

Down's syndrome 111, 582

Doyle, E. F. 302*

Draffan, H. 54*

Draper, D. $406^{*}$

Drash, A. L. 330*

Dray, F. 322*

Drummond, K. N. 408*, 410*, 412*, 414*

Dubrawsky, C. 429 *

Ducharme, J. R. 323*

Duckett, G. 332*

Duczyńska, N. 65 *

Duffy, T. E. $424^{*}$

Dulac, H. 46*

Dumars, K. W. 331 *

Dumpit, F. $430^{*}$

Dupon, C. $332 *$

Dupree, E. 364*

Durand, P. 61*, 346*

Dwarfism 71

Dweck, H. $386^{*}, 400 *, 404 \%, 407$ *

Earle, N. R. $317 *$

Earon, J. $413 *$

Ebert, P. A. 290*

Edelmann, C. M., Jr. 408*, 411*, 413*

Edwards, N. K. $405 *$, 408*

Ehrlich, R. $331 *$

Eisner, G. M. 414*

Eitzman, D. V. 425*

Elders, M. J. $324 *, 420 *$

Elkins, R. 305*

Eller, E. A. 374*

Eller, J. J. 374*

Elliott, R. B. 427 *

Fisas, L. J. 382*

Embil, J. A. 394*

Emery, E. S. 313\%

Emich, J. P. 319*

Emmanouilides, G. C. $396^{*}$, 401*

Engel, R. R. 292*

Engelbart, S. 588

Engle, M. A. 290*

Environment 888

Epilepsy 914

Epincphrine 787, 983 
Epithelium 607

Epstein, C. J. 346*

Erenberg, A. $311^{*}, 325^{*}, 401 *, 870$

Erhard, D. 415*

Erickson, R. P. $346^{*}$

Eriksen, L. 289*

Eriksson, M. 317*, 319*

Erythrocytes 954

Escobedo, M. B. 431*

Esperanza, R. F. 427*

Ester, L. 335*

Estcrly, J. R. 339*

Estrada, M. 401*

Estradiol 826, 901, 948

Estradiol-17, $\beta 139$

Estrone 826, 901

Etteldorf, J. N. 325*

Ettenger, R. 832

Euglobins 220

Evans, A. E. 357 *

Evans, D. A. 302*

Evans, H. E. 320*

Exelby, P. 361*

Fabry's discase 684

Faiman, C. $306 *, 948$

Fainney, A. $391^{*}$

Falchuk, Z. M. 336*

Fallat, R. 383*, 387*

Fällström, S. P. 53*

Family dynamics 520

Fanaroff, A. A. 396*, 607

Fanconi, A. 47\%

lang, V. S. $332^{*}, 414^{*}$

Faraj, B. A. 321*

Farias, N. E. 541

Farrell, P. M. 308*, 311*, 313*, 427*

Farzanch, I. 431*

Fass, R. 360*

Fceney, M. 410*

Fchr, P. 318*

Feig, S. A. $355^{*}$

Fcigin, R. D. 500

Fcketc, M. 53*

Iclclman, R. A. 4I7*

Feldman, W. 297*

Fellers, F. X. 417*

Feng, K. K. 373*

Fenner, A. 174

Fenton, L. J. 323*

Fcrrara, A. 401*

Ferritin 954

Fctal hypcrcoagulability 670

Fetus $1,20,75,95,126,132,192,509,527$, $675,696,706,719,769,794,812,826,870$ $878,908,937$

Fctus, rhesus 787

Fevrier, A. 375*

Fibroblast(s) 13, 645

Ficin 684

Ficlds, D. 311*
Filer, L. J., Jx. 386*

Fine, R. N. $45^{*}, 414^{*}, 712$

Finegold, M. 302*

Fingerprints 215

Fink, C. W. 71

Finkelstcin, J. W. 323*, 328*

Finland, M. 320*

Yinncgan, L. P. 292*, 319*

Fischbein, C. 303 *

Fischer, E. G. 303*

Fiser, R. H. $311^{*}, 315^{*}, 383^{*}, 407^{*}$

Fisher, D. A. $311^{*}, 325^{*}, 333^{*}, 383^{*}, 401 *$, $407^{*}, 870$

Fisher, D. E. 32I*, 405*, 418*, 427*

Fisher, M. 399*, 400*

Fishman, G. 422*

Fitzhardinge, P. $311^{*}$

Fleidner, T. M. 48*

Fleisher, L. D. 386 *

Flcming, D. $396^{*}$

Fletcher, 13. D. $430^{*}$

Florman, A. L. 373*

Flynn, M. A. 328*

lilynn, ㅅ. M. 392*

Folate 527

Folic acid 729

Folland, D. 322*

Follicle-stimulating hormone 948

Iones, D. W. 367*

Forman, E. 289*

Forman, T. F. 345*

Forster, R. E. 291*, 401*, 427*

Forticr, N. 350*

Fosbrooke, A. $54^{*}$

Fowler, R. L. 428*

Fox, E. N. 372*

Fraccaro, M. 66*

Franchini, I. 62*

Frankel, A. S. 368*

Frankcuburg, W. K. 294*

Franklin, G. 420 *

Frantz, T. A. 395* $402^{*}$ *

Franz, K. 46*

Frascr, D. 324*

Frasicr, S. D. $328^{*}, 331^{*}$

Iraumeni, J. F. 620

Freclman, M. H. 331*, 356*

Frec fatty acids 192

Freeman, A. I. 353*

French, J. H. 386*

Frias, J. L. $387 \%, 395 \%$

Frick, O. $365^{*}$

Friday, G. A. 339*

Friedman, A. S. 295*

Friedman, C. J. 295*

Friedman, E. 410*

Friedman, M. 353*

Friclman, S. 311*, 360*

Friedman, S. B. $295^{*}$

Friedman, W. F. 301*

Triesen, H. G. 534
Frommes, S. 389*

Fructose 500

Fuccillo, D. A. 372*

Fuchs, M. 294*

$\alpha$-lucosidase 751

Fudenberg, H. H. 362*, 367*

Fumcro, S. 60*

Fussgängcr, R. 100

Fyler, D. C. 30 I* $^{*}$

Gaburro, 1). 57*, 198

Gacta, J. F. 353*

Gagnadoux, M. F. 67*

Gajl-Peczalska, K. J. 349*

$\alpha$-Galactosidase 684

$\beta$-Galactosidase 751

Galant, S. P. $427^{*}$

Galjaard, H. 56*

Gall, D. G. 337*

Galvez, M. B. 411*

Ganaway, R. L. 367*

Gangarosa, E. J. 376*

Gangliosidc $N$-acetyl neuraminic acid 812

Gangliosidosis Type 1812

Garbaccio, A. 413*

Garcia, A. 402*

Garcia, O. L. 426 *

Garcia, S. J. 318*, 398*

Gardner, L. I. 328*, 330*, 344*

Gardner, P. $413^{*}$

Garnica, A. 387*

Garrison, R. D. 425*

Garry, P. J. 296*, 359*, 392*

Gartner, L. M. 119, 320*, 338*

Garvin, A. J. 291*

Garvin, W. F. 298*

Gates, A. H. 319*

Gatti, R. 61*

Gaull, G. E. $58^{*}, 307^{*}, 316^{*}, 347^{*}, 386^{*}$, 527,908

Gauthicr, B. 410*, 415*

Gay, G. 350\%

$\mathrm{G}$ bands 39

Gehlbach, S. H. 392*

Gelband, H. 303*, 426*

Gelchrter, 'T. D. 381*

Gelfand, E. W. $367^{*}$

Geller, G. R. 355*

Genel, M. 299*, 332*, 818

Genes 5

Genetic diseasc 220, $500,645,684,700,757$, 994.

Genetic disorder 14.9

Genscher, U. 62*

Gentz, J. 58 **

Gcorges, M. 385*

Gcrald, P. 344*

Gerety, R. J. 370*

Gerhardt, T. O. 396*, 402*

Gcrmuth, F. G. 413*

Gcrshon, A. 373* 
Gersony, W. M. 299*, 300*

Gessner, I. H. 311*

Ghadimi, H. 161, 169, 387*, 398*

Ghadimi, R. 390*

Gharib, M. 413*

Ghatak, N. R. 386*

Giacoia, G. P. 317*, 320*

Giangiacomo, J. 413*

Giannopoulos, G. 310*

Giliberti, P. 60*

Gilkerson, E. 351*

Gill, F. M. $311^{*}$

Gillespic, D. ‥ 424*

Gilman, P. A. 357*

Ginsberg-Fellner, F. 291*

Giovannini, M. 60*

Girard, J. 62*

Giroud, C. J. P. 308*, 329*

Gitlin, D. 290*, 339*, 368*

Gitlin, J. P. 290*

Gitzclmann, R. 63*

Given, G. Z. 415*

Glade, P. R. 366*, 369*

Glader, B. $350 *$

Glaser, B. 397*

Glaser, J. H. 342*

Glasgow, L. A. 371*, 378*

Glass, L. 320*

Glauser, E. M. 423*

Glauser, S. C. $423^{*}$

Glick, T. 423*

Gloria, F. 54 *

Glorieux, F. 387*

Glucagon 184, 500, 983

Gluck, L. 406* $407 *$, 429*

Gluconeogenesis 184, 500

GIucose 100, 794

Glucose metabolism 691

Glucose 6-phosphate dehydrogenase 75

Glueck, C. J. 312* 383*, 387*, 406*

$\gamma$-Glutamyltranspeptidase 95

Glycine 945

Glycogen 739, 745, 769

Glycogenosis 745

Glycogenosis, type II 739

Glycogenosis, type III 739

Glycolipid 560

Glycosaminoglycans 965

Gnarra, D. J. 351*

Goad, W. 344*

Godard, C. $47^{*}$

Godman, M. J. 392*

Goh, K. 351*

Gold, E. 375*, 376*

Gold, R. 370*

Gold, R. J. M. 342*

Goldberg, B. 402*

Goldberg, H. 119

Goldberg, M. F. 422*

Goldblum, R. M. 363*, 364*, 367*

Goldfischer, S. $386^{*}$
Goldman, A. S. 323*, 332*

Goldman, A. S. $363^{*}, 364^{*}, 367^{*}$

Goldman, H. I. 149, 337*, 418*

Goldman, J. S. $418^{*}$

Goldring, D. 306* , 431*

Goldschneider, I. 370*

Goldsmith, D. I. 411*

Goldsmith, R. S. 485

Goldstein, G. 418*

Goldstein, M. N. 358*

Goldstein, R. 332*

Goldstein, S. 313*

Gonadotropin 948

Gong, B. T. 423*

Good, R. A. 349*

Gooden, D. S. 401*

Goodman, J. R. 337*

Goodwin, J. F. 390*

Gootman, N. 303*

Gootman, P. M. 303*

Gordon, M. 295*

Goto, H. 425*

Gotschlich, E. C. 370*

Gouw, W. L. M. 863

Governa, M. 62*

Gower, D. B. 328*

Grach, J. L. 426*

Graham, F. K. 419*

Graham, G. G. 338*

Graham, T. P., Jr. 318*

Gralnick, H. 353*

Grand, R. 335*

Granelli, S. F. 352*

Grassy, R. G. 402*

Graven, S. N. 395*, 402*

Graziani, L. J. $420 *$

Green, M. 295*

Green, O. C. $328 *$

Greene, H. L. $335 *, 337 *$

Greener, Y. 357*, 937

Greenwood, M. 375*

Gregoriou, M. 67*

Gregory, T. 328*, 330*

Greifer, I. 408*

Griffin, B. E. 398*, 431*

Griggs, R. C. $424 *$

Grimes, E. R. 375*

Griscom, N. T. 400*, 424*

Grishaver, M. A. 343*

Griswold, W. R. 366*, 410*

Grodzka, Z. 65*

Grose, C. 370*

Groshong, T. 368*

Gross, E. 798

Gross, I. 308*

Gross, S. 404*

Gross, S. 296*

Grover, W. 832

Growth and development 719

Growth hormone 71, 527

Growth rate 485
Growth retardation 883

Grumbach, M. M. 290*, 291*

Grupe, W. E. 413*

Grushkin, C. M. 414*, 712

Gruskin, A. B. 412*, 414*, 832

Guild, P. 295*

Gunay, U. 356*

Gunn, T. 326*

Gunther, S. 354*

Guntheroth, W. G. 428*

Guroff, G. 322*

Gutberlet, R. L. 312*

Guthrie, R. 64*

Gutman, A. 66*

Gutman, L. T. 372*

Guyda, H. J. 534

Gyurkovits, K. 63*

Haas, R. J. 48*

Hachey, D. L. 34I*

Haddad, J. G. 312*

Haddad, Z. H. 367*

Haemophilus influenzae type $b 103$

Hagan, A. D. 301*

Hagenfeldt, L. 60*

Haggerty, R. J. 297*, 520

Haghbin, M. 352*

Hahn, P. 75, 308*, 381*

Hales, B. 320*

Hall, C. W. $343 *$

Hall, C. St.-G. 332*

Hall, D. 415*

Hallal, R. 60*

Hallman, M. 923

Halscy, J. H., Jr. 420*

Halvorsen, K. 62*

Halvorsen, S. 62*

Hambidge, M. 337*

Hamburger, R. N. 368*

Hamerton, J. L. 347*

Hamid, J. 883

Hamilton, J. R. $337^{*}$

Hamly, C.-A. 387*

Handzel, Z. T. 370*

Hänggi, C. 54*

Hanley, W. B. 383*

Hann, S. 363*, 368*, 375*

Hans, M. 67*

Hanscll, J. 336*

Hanson, P. A. 419*

Hardy, G. E., Jr. 289*

Hardy, M. 4I1*

Harevy, D. 47*

Harh, J. Y. 312*

Harper, R. G. 402*, 403* , 408*

Harris, G. B. C. $400 *$

Harris, M. B. 352*

Harris, S. L. 293*

Harris, T. C. $404 *$

Harrison, E. G., Jr. 429*

Harrison, V. C. $398^{*}$ 
Harrod, E. K. 601

Hart, M. C. $355^{*}, 398^{*}, 431^{*}$

Hart, Z. H. 390*

Hartlage, L. C. 295*, 345*

Hartlage, P. L. 295*

Hartmann, A. F, Jr. 306*

Harvey, J. S., Jx. 367*

Hass, G. 301*

Hastings, C. 696

Hastreiter, A. 322*

Hatch, M. 376*

Hathaway, P. 220

Hathaway, W. E. $361^{*}, 396 * 670$

Hattwick, M. 376*

Hauhart, R. E. $312^{*}, 388^{*}, 691$

Havlicek, V. 20

Havron, W. S. 370*

Hayck, A. 28, 324*

Hayes, F. A. $355^{*}$

Hayes, W. 408*

Hayles, A. B. $325^{*}, 326^{*}, 328^{*}$

Haymond, M. W. 381*, 384*, 388*, 500

Haymovits, A. 393*

Haynes, R. E. 392*

Hayslett, J. P. 290*, 417*

Hazlewood, C. F. 331*

Health care 520

Heart 494, 931

Heat loss 888

Hecse, H. deV. 398*

Heinze, E. 100

Hellerstein, S. 356*

Hellman, L. 323*, 328*

Hellwege, H. H. 61*

Helmrath, T. A. $309 *$, 310*

HeIson, L. 388*

Hematocrit 937

Hemoglobin 954

Hendley, J. O. $375^{*}$

Hendren, W. H. 327*

Henley, W. L. 365*

Hexning, L. B. 293*

Hensen, S. A. 372*

Hepner, R. 295*

Herberman, R. B. 292*

Herbert, J. 364*, 380*

Heriot, J. T. 293*

Herman, S. 50*

Herman, Y. F. 337*

Hernandez, A. 306*

Hernandez, R. 61*

Herrick, N. 729

Herrin, J. T. $423 *$

Herrin, W. 340*

Herrmann, K. I. 289*

Hers, H. G. 59*

Herschkowitz, N. 48*, 49*, 52*

Hersh, C. B. $312^{*}, 406^{*}$

Hertzberg, L. J. 294*

Herz, F. 204

Hessler, J. R. 425*
Hevizy, M. 370*

Hewitt, J. R. 313*

Hickman, R. 413*

Higer, R. W. 428*

Higgins, G. R. 361*

Higurashi, M. 582

Hill, H. R. 371*

Hillman, L. S. 312*

Hillman, R. E. $384 *$, 388*, 945

Hilmar, N. 298*

Hilton, J. D. $356^{*}$

Hilty, M. D. 388*, 392*

Hindlimb 794

Hiner, L. 412*

Hirano, A. 386*

Hirschhorn, K. 220, 224, 343*, 344*, 386*, 958

Hirsh, J. 358*

Hirshaut, Y. 378*

Hitchcock, E. S. 421*

Ho, C.-K. 342*

Hobbs, J. R. 317 *

Hobcl, C. J. 401*, 407*

Hochman, H. 393*

Hochmuth, R. M. 362*

Hodes, H. L. 370*

Hodge, J. S. 53*

Hodgman, J. E. $397 *$, 424*

Hodson, W. A. 302*

Hocnicke, H. 174

Hoffman, B. F. 300*

Hoffman, D. E. 42I*

Hoffman, D. R. $367^{*}$

Hoffman, J. X. E. 429*

Hoffman, L. I. 428*

Holick, M. 387*

Holland, P. 335*, 375*

Hollander, M. 119

Holliday, M. A. $411^{*}, 414^{*}, 415^{*}, 428^{*}$

Hollister, D. W. 343*, 348*

Holowach-Thurston, J. 312*, 388*, 691

Holsapplc, R. 296*

Holtzapple, P. G. 337*, 339*, 818

Holtzman, N. A. 382*

Holzman, R. S. $378^{*}$

Hommes, F. A. $52 * 616,863$

Homocyst(e)inc 527

Homocystinuria 224, 645

Hon, E. H. 404*, 405*

Hong, R. 363*

Honig, G. R. $307^{*}, 347 *, 351^{*}, 356 *$

Hook, E. B. 312*

Horger, E. O., III 291*

Horn, K. 51*

Horowitz, S. 363

Horwitz, S. J. $377^{*}$

Hosmer, M. E. 377*

Hotchkiss, J. 826

Houslex, M. E. 601

Houston, I. B. 65*

Hoversten, G. H. 347*
Howatt, W. F. 321*

Hoyer, J. R. 292*

Hrbck, A. 5 I * $^{*}$

Hruby, M. A. 356*

Hsia, Y. E. 290*, 334*, 384*, 388*

Hsu, L. Y. F. 343* 344*

Huang, N. N. 428*

Huang, T. L. 340*

Hubbcll, J. P. 305*

Huber, G. L. 430 *

Huber, J. 367*

Hue, L. 59*

Huff, D. S. $363^{*}, 375^{*}, 832$

Hug, G. 389*

Hughes, E. R. 324*, 420*

Hughes, W. T. $370 *$, 374*

Huijing, F. 343*

Huisjes, H. J. 863

Hume, D. 358*

Humphrey, A. $354^{*}$

Hundley, J. R. $347 *$

Hunt, C. E. $292 *, 304^{*}, 428 *$

Hunt, P. A. 421*

Hurley, R. M. 408*, 410*

Hurwitz, A. $340 *$

Hurwitz, E. 675

Hurwitz, I. 293*

Hutcheson, M. W. 345*

Hutchinson, D. L. 368*

Hyaline membrane disease 82,638

Hydrogen ion excretion 712

2.5-Hydroxycholccalcifcrol 914

Hydroxyproline 818

Hydroxyputrescine 994

Hyman, C. B. 361*

Hyperbilirubinemia 100

Ixyperlysinemia 994

Hyperthyroidism 28

Hypoglyccmia 500, 945

Hypoxanthinc-guanine transferase 729

Hypoxia 569

Hypusine 994

Iannetta, A. 374*

I-cell disease 13, 560

Idiopathic respiratory distress syndrome 670

Igo, R. P. $371 *$

Ikossi, M. G. 691

Illness 520

Iminoglycinuria 818

Immunity 103, 778, 883

Immunoglobulin(s) 103, 541, 659, 675

Inamclar, S. 408*

Infant, premature 638

Infants 161

Ingram, D. L. $369 *$, 378*

Inoue, S. 349*

Insulin 100, 745, 787

Intestine 95 
Iodothyronine 870 Ionized calcium $48 \tilde{5}$ Iosub, S. 294*, 420* Isenberg, J. N. 389* Isoleucine 149,945 Israili, Z. H. 321* Isselbacher, K. J. 334* Ives, E. J. 345* Ivler, D. $318^{*}$

Jacinto, E. Y. 412* Jackson, C. E. $345^{*}$ Jackson, R. L. 391* Jacob, H. S. 355* Jacobs, L. S. 333* Jacobsen, J. B. 53*, 334*, 397* Jacobson, C. 291* Jaenicke, R. 352* Jaimovich, C. $414 *$ Jain, S. K. $305^{*}$ James, F. W. 299* James, L. S. 299*, 397* Jamicson, A. M. 313*, 965 Jancway, C. A. 853 Jansen, V. 192 Jasper, H. G. 307* Javitt, N. B. 119 Jawad, J. 375* Jenkins, D. C. 400 * Jensen, A. H.-B. 638 Jéquier, E. 888 Jéquier, J. C. $323^{*}$ Jesse, M. J. $426^{*}$ Jirari, C. G. 421 * Job, J. C. $385^{*}$ Johnson, D. G. 419* Johnson, D. H. 303* Johnson, J. D. 334* Johnson, J. WV. C. 402* Johnson, L. 403\% Johnson, R. C. $306^{*}$ Johnson, S. 410* Johnston, C. L. $360^{*}$ Johnston, M. $358^{*}$ Johnston, R. B., Jr. 364* Jones, E. 375* Jones, E. M. 312*, 388*, 691 Jones, H. S. $385^{*}$ Jones, O. W. $307 * 343 *$ Jose, P. A. 414* Josso, F. 638 Josso, న. 322* Jouja, V. 659 Jourdian, G. W. 393* Jowscy, J. 757 Juan, C. $327 * 384 *$ Juberg, R. C. 347 * Jubiz, W. 32I* Just, M. 61 *

Kaback, M. M. 55*, 343*, 812 Kachra, Z. 342*
Käckell, Y. 53*

Kafatos, A. G, 370*

Kaiser, D. 63*

Kaiser, H. J. 588

Kalavsky, S. M. 421*

Kalhan, S. 381*

Kallen, R. J. 414*

Kamali, H. 332*

Kamensky, E. 348*, 389*, 392*

Kandall, S. R. 320*, 356*

Kaplan, B. S. 414*

Kaplan, E. L. 204, 372*, 375*

Kaplan, S. 299*, 300*, 301*

Kaplan, S. A. $325 *$, 424*

Kaplan, S. L. 290*, 29I*

Kapp, J. P. 320*

Karayalcin, G. 353*, 357*

Karjoo, M. 337*

Karl, I. E. 381*, 388*, 500

Karlberg, P. 51*

Karon, M. 310*, 352*, 361*

Kashgaxian, M. 290*, 417*

Kassner, E. G. 403*

Kattwinkel, J. 396*

Katz, K. S. 293*

Katz, L. 420*

Katz, M. 53*

Katz, S. 354*

Kaufman, D. 13. 364*, 410*

Kaufman, I. 4I8*

Kaufman, M. 332*

Kaufman, R. 348*

Kawahara, F. S. 389*

Kayden, H. J. 192

Kaye, C. I. 346*

Kazazian, H. H., Jr. 343*

Kazemi, H. 423*

Kazemi, M. 320*, 376*

Keating, J. P. 388*

Keidel, W. N. 429*

Kcim, H. 422*

Keitges, J. $414 *$

Keith, J. D. 305*, 392*

Kekomäki, M. 309*

Kelch, R. P. 326*

Keller, M. 351*

Kelly, M. H. 337*

Kempc, C. H. 293*

Kempe, R. S. 293*

Kennell, J. 296*

Kenny, F. M. 326*, 328* 329*, 390*, 826

Kenny, J. 329*

Kent, G. $425^{*}$

Kenyon, K. R. 560

Kern, E. 378*

Kerr-Grant, D. $377^{*}$

Kersey, J. H. 349*

Kershnar, A. K. 329*

Ketotic hypoglycemia 983

Khachadurian, A. K. 387*, 389*

Khan, A. U. 294*

Khurana, N. $416^{*}$
Khuri-Bulos, N. 376*

Kidd, B. S. L. 300*

Kidney 553, 818, 878

Kidney transplantation 712

Kienstra, R. A. 413*

Kicrnat, J. F. 374*

Killam, A. P. 139

Kim, D. S. $369^{*}$

Kim, H. J. 344*

Kim, H. W. 373*

Kim, K. Y. 353*, 357*

Kim, M. H. 332*

Kim, S. H. 327*

Kim, Y. J. 291*

Kind, H. P. 324*

Kindler, P. 62*

King, K. 329*

Kinney, T. 361*

Kirby, L. 75

Kirby, L. T. 308*

Kirkpatrick, C. H. 364*

Kisker, C. T. $358^{*}$

Kissane, J. 306*

Kistenmacher, M. L. 348*, 368*

Kitabchi, A. E. $325 *$

Kitz, R. 300*

Kjellmer, I. 51*

Kjellstrand, C. M. 292*

Klagsbrun, M. 313*

Klain, D. B. $396^{*}, 403 *$

KIaus, M. 296*, 396*

Klebanoff, S. J. 371*

Klein, G. 329*

Klein, J. O. 320*

Klein, M. 398*

Klein, P. D. $340^{*}, 341 *$

Klein, R. 397*

Kleinberg, F. 403*

Kleincrman, J. I. 607

Klcinman, L. I. 403*, 412*

Klemperer, M. R. 351*

Kline, E. 380*

Klotz, D. 398*

Kniker, W. T. 367*

Knittle, J. L. 291*

Knobloch, H. 296*

Knorr, D. 901

Koch, H. F. 396*

Koch, K. 417*

Koch, R. 59*, 391*

Kochen, J. A. 357*, 358*, 937

Koenigsberger, M. R. 421*

Koffler, H. 323*

Kogut, M. D. 329*

Kohl, S. G. 289*

Kohlschütter, A. 49*

Kohne, E. 66*

Kohrman, A. F. 575

Kok, E. 119

Kollmeyer, K. R. 403*

Komp, D. M. $357^{*}, 376^{*}$ 
Koo, E. L. 403*

Koo, J. 314*, 415*

Kooh, S. W. 324*

Kopel, F. B. 220

Koprowski, H. 342*

Korth-Schutz, S. 411*

Koss, M. N. 366*

Kotas, R. V. 401*, 429*

Kouri, R. 310*

Kowarski, A. 509

Kozinn, P. J. 378*

Kramer, H. 417*

Kramcr, M. S. $357^{*}$

Krasncr, J. 308*, 317*, 334*

Krasula, R. 322*

Krauer, B. 54*

Krauss, A. N. 82, 396*, 403*, 569

Kravath, R. E. 296*, 357*

Kream, J. 328*

Kreil, E. 300*

Krempien, B. 46*

Kretschmer, R. R. 371*

Krieger, I. 313*, 329*, 390*

Krivit, W. 355 *

Kron, R. E. 292*

Krongrad, E. 299*, 300*

Krovetz, L. J. 303*

Krugman, R. D. 289*, 370*

Krugman, S. 846

Kruse, H.-P. 45*

Kuhlencordt, F. 45*

Kulczycki, L. 429*

Kulin, H. E. 323*

Kulovich, M. V. 354*, 429*

Kulvinskas, C. $416^{*}$

Kumar, M. L. 376*

Kumar, S. 161, 390*

Kündig, H. 432*

Kunos, G. 317*

Kushner, I. 403*

Kwon, O. B. 305*

Labcdz, R. 883

LaBrosse, E. H. 357*

Lachman, R. 348*

Lactate 832

Lacssig, R. 729

Lafave, H. 296*

Laffin, R. J. 367*

Lahey, M. E. 419*

Laird, W. P. 303*

Lakeman, A. W. 379*

Lamm, P. 149, 390*

Lampe, J. 298*

Lampcrt, P. 407*

Lampkin, B. C. 355*

Lancaster, G. 390*

Land, V. J. 358*

Landaw, S. A. $356 *$

Lang, R. 333*

Lange, K. 417 *
Langer, H. 429*

Langley, C. E. $343^{*}$

Lanman, J. T. 289*

La Pointe, D. 336*

Laraya-Cuasay, L. 428*

Larrier, L. 373*

Larson, R. M. 326*

Larsson, A. 60*

Lasalle, R. 335*

Latorre, H. 329*, 390*

Lattos, D. $363^{*}$

Lau, H. L. 402*

Laucr, B. 380*

Laver, M. B. 301*

Lawlor, G. J., Jr. 364*, 369*

Lawton, A. R. 364*

Lazerson, J. 361*

Lead 937

Leakc, R. D. 381*

Leavitt, L. A. 419*

LebenthaI, E. 337*

Leblanc, W. 321*

Leboeuf, G. 323*

Lecithin 632

Ledlcy, R. S. 39

Lee, C. L. Y. 349*

Lce, H. $351^{*}$

Lee, J. C. $30 I^{*}$

Lee, J. J. 336*

Lee, K. 338*

Lee, S. J. 289*

Lee, T.Y. $313 *, 965$

Leib, U. 54*

Leigh's encephalomyelopathy 616

Leigh's syndrome (encephalomyclitis) 832

Leikin, S. L. 368*

Leisti, S. 66*

Leititis, J. 58*

Lenard, H. G. 51*, 588

Lenoir, M. A. 368*

Lentz, G. 295*

Leonard, A. S. 428*

LePourhict, A. 292*, 353*

Lepow, M. L. 370*

Leppla, W. $58^{*}$

Lerer, R. J. 421*

Lesch-Nyhan syndrome 729

Lester, R. 292*, 341*

Letarte, J. $323^{*}$

Leuba, V. $387^{*}$

Leukemia 620

Ieukemia, acute lymphocytic 132

Ieukocytes 224

Leung, P. 400*

Leupold, D. 53*

Leupold, E. $66^{*}$

Leventhal, B. G. 292*, 353*

Levi, D, F. 417*

Levi, J. 89

Levin, A. S. $367^{*}$

Levin, B. 728
Levin, S. 675

Levinc, L. S. $331 *$, 409*

Levine, M. M. 376*

Levine, O. R. 931

Levinson, H. 331*, 425*, 429*

Levitsky, L. L. $418^{*}$

Lcvitt, M. D. 292*

Levitz, M. 192

Levy, G. 320*

Levy, H. L. 390*

Levy, R. L. 363*

Lewin, E. B. $320 *$, 430*

Lewy, J. E. 409*, 411*, 412*, 415*, 553

Lewy, P. R. $415^{*}$

Li, Y.-T. 684

Librik, L. 331 *

Lie, S. O. 13,57 *

Liebman, J. 302*

Liebman, O. B. $418 *$

Licbman, R. 294*

Liebman, W. M. 338*

Liedholm, M. 51*, 53*

Lifshitz, F. 340*, 341*, 391*, 914

Light, I. J. $323^{*}, 403^{*}, 404^{*}$

Lightbody, J. J. 363*

Isilis, M. 369*

Lim, H. H. 402*

Lim, W. N. 378*

Limbcck, G. A. 331*

Linarelli, L. G. $329 *, 330 *, 878$

Lind, J. 304*

Lindall, A. W. 323*

Lindbach, T. 402*

Lindblad, 13. 342*

Linde, L. M. 304*

Linder, D. 391*

Lindmark, D. G. 601

Lindsay, M. $351 *$

Lindstrom, D. 426*, 431*

Linoleic acid 192

Linsao, L. 383*

Linshaw, M. 832

Lione, A. P. 418*, 422*

Lipolysis 575

Lippc, B. M. 325*, 423*

Lipsitz, P. J. 404*

Lischner, H. W. 363*, 368*, 375*

Lister, G., Jr. 429*

Litt, I. F. 334*

Litt, M. 292*

Litwin, S. B. 301*

Liu, C. $373^{*}$

Lively, W. M. 338*

Liver 1, 75, 494, 560, 863, 923

Lloyd-Still, J. D. 293*

Lockard, W. 404*, 407*

Lodgc, A. 420*

Lodinová, R. 659

Loeb, H. 57*

Loirat, C. $45^{*}$

Lonsdale, D. $421 *$ 
Lopata, M. A. 310*

Lopez-Osuna, M. $371^{*}$

Lord, R. A. 363*, 364*

Lorentz, W. B., Jr. 409*

Lorincz, A. E. 343*

Lovelace, R. E. 421*

Low birth weight 184

Lowry, M. 381*

Lu, C. $327 *, 385^{*}$

Lu, T. $306^{*}$

Lubawy, C. $375^{*}$

Lubin, A. H. 296*, 359*, 392*

Lubin, B. H. $303^{*}, 337 *$, 354*

Lubin, H. 297*

Lubs, H. A. 39

Lucarielli, P. 54 *

Lucas, M. $343^{*}$

Lucas, R. V., Jr. 300*, 304*

Lucey, J. F. 313*, 397*

Luit-de Haan, G. 616, 863

Luke, K.-H. 358*

Lum, R. K. 706

Lund, H. T. $53 *$

Lundborg, P. 53*

Lung(s) 82, 632

Lusher, J. M. 353*

Luteinizing hormone 948

Lyles, R. L. 357*

Lyles, T. O. $417 *$

Lymphocytes 582,700

Lyon, I. C. T. $65 *$

Lyons, E. C. $409 *$

Lysine 994

Lysosomal phosphatase 224

Lysosome(s) 13, 560, 751, 798

Lysozyme 659

Mabry, C. C. $345^{*}$

Macagno, F. 47*

MacDonell, R. C., Jr. 411*, 414*

MacGillivray, M. H. 325*, 330*, 366*

Maclaren, N. 391*, 914

Maclean, W. C., Jr. 338*

MacMillan, D. R. $324 *, 719$

MacMurray, S. B. 430*

MacQucen, D. Mr. $372 *$

Maddaian, V. T. $316^{*}, 336^{*}, 385^{*}, 394^{*}$

Madhavan, S. $305^{*}, 431 *$

Madigan, P. M. $390 *$

Magnesium 485

Mahasandana, C. $396 * 670$

Mahoney, M. J. 342*, 384*

Maiden, N. 295*

Main, C. A. $352 *$

Makino, S. 343*

Makkcr, S. P. 313*

Makowski, E. L. 139, 309*, 314*, 396*, 794

Malekzadeh, M. H. 414*, 712

Malik, A. B. 300

Malnutrition $494,778,883$

Maloof, F. 28, 327*
Maltz, H. E. $415^{*}$

Mamer, O. A. 149,390 *

Mammary gland 139

Mamunes, P. 421*

Manasek, F. J. 306*

Mandalenaki, K. 362*

Mandell, R. 382*

Manganiello, V. 391*

Mangiarotti-Marchi, M. A. 63*

Mangos, J. A. 336*, 338*

$\alpha$-Mannosidase $75 \mathrm{I}$

Mansell, A. 425*, 429*

Mantel, N. 132

Mapa, H. C. $42 \mathrm{I}^{*}$

Maple syrup urine disease 500

Marchi, A. G. 63*

Marcy, S. M. 344*

Marks, J. F. 71, 391*

Marks, M. エ. $320 * 376^{*}$

Markwell, M. A. K. 310*

Marliss, E. B. $59 * 383^{*}, 983$

Maron, B. J. 303*

Marr, T. 415*

Marshall, J. 349*

Marshall, R. E. 431*

Martin, H. L. 379*

Martincz, M. 49*

Mascia, A. V. 627

Mason, E. O. $365^{*}$

Mason, R. G. 307*, 351*, 356*

Massimo, L. 62*

Mastella, G. 63*

Matalon, R. 384*, 684

Matalon, S. 428*

Matanic, B. 306*

Matheny, A. P., Jr. 324*, 719

Mathies, A. W. 318*

Mathieu, H. 46*, 67*

Matsaniotis, N. 55*, 67*

Matthews, L. W. 340*, 430*, 607

Matthieu, J.-M. 49*

Mattson, J. 410*

Mauer, A. M. $350 *$, 355*

Mauer, S. M. 292*

Maurer, H. M. 354*, 358*, 359*, 360*, 406*

Maurer, H. S. $351^{*}$

May, A. 351*

Mayberry, W. E. 706

McAlister, W. 348*

McAnulty, P. A. 778

McAtee, P. 298*

McCabe, J. 290*

McCall, C. E. 364*

McCall, J. T. 485

McCarthy, D. J. 350*

McClenathan, J. 429*

McClure, P. D. $356^{*}$

McConnachie, P. R. 371*

McCormick, B. 322*

McDaniel, J. 354*

McDonagh, A. F. 352*
McEIroy, G. L. 338*

McEwan, S. N. 406*

McFarlane, H. 883

McInnes, R. 409*

McIntosh, R. M. 366*, 410*

McIntosh, S. 343*, 358*, 359*

McKenna, J. 399*, 400*

McKnight, R. $306^{*}$

McKusick, V. A. 57*, 348*

McLain, C. R. 389*

McLaughlin, G. W. 396*, 425*

McLean, F. H. 408*

McLeod, A. G. W. 343*

McNamara, H. 319*

McNatt, L. M. 324*

McNatt, M. L. $420^{*}$

McSherry, N. R. 336*, 338*

McVicar, M. 410*, 415*

McWilliams, N. B. 359*, 406*

Mead, J. F. 379*

Meara, P. A. $385 *$

Measles 582

Medina, J. V. 414*

Medulla 20

Mehls, O. $46^{*}$

Mehta, S. 317*

Melchior, J. C. $57 *$

Melhorn, D. K. 359*, 404*

Mellin, G. W. 377*

Mellins, R. B. 627

Mellman, W. J. 348*, 394*, 739, 745

Mendecki, J. 358*

Mendicini, M. 50*

Mendilaharzu, H. 330*

Meng, C. C. L. $304^{*}$

Meng, H. C. 405*, 431*

Menkes, J. H. 311*

Menser, M. A. 215

Mental retardation 601, 700

Mercado, H. 302*

Mercer, R. D. 421*

Merenstein, G. B. 335*, 337*

Merk, J. 403*

Merker, C. $300^{*}$

Merrick, S. 39

Meschia, G. 139, 309*, 314*, 794

Metabolic regulation 794

Metaphase 39

Methionine 908

$\alpha$-Methylacetoacetate 149

1-Methylhistidine 601

$\alpha$-Methyl- $\beta$-hydroxybutyrate 149

Methyltransferase 527

Metzger, B. E. 405*

Meuwissen, H. J. 362*, 367*, 369*

Meyer, B. P. 398*, 404*, 431*

Meyer, H. M., Jr. 289*, 370*

Meyer, R. A. $300^{*}$

Meyer, V. W. 304*

Meyermann, R. 53*

Meyers, G. 380* 
Michacl, A. F. 292*

Michaels, R. H. 377*

Microspheres, radioactive 139

Midha, R. 428*

Migeon, C. J. 509

Mikkelsen, E. J. 296*

Miles, V. N. 306*

Millar, E. A. 361*

Miller, C. S. 751

Miller, D. L. $339^{*}, 368^{*}$

Miller, H. C. $400 *$

Miller, J. R. $368^{*}$

Miller, K. 348*, 394*, 812

Miller, L. D. 291*, 401*

Miller, M. E., 365*, 368*, 416*

Miller, R. 358*

Millman, I. 365*

Milman, L. 294*

Milner, R. D. G. 53*

Milstead, R. 386*, 400*

Milunsky, A. 313*

Mims, L. C. $401^{*}$

Minkorwski, A. 638

Minnefor, A. B. 359*

Minuchin, S. 294*

Mirkin, B. L. 318*, 417*

Mitochondria 616,923

Mize, C. E. 338*, 391*

Mockrin, L. D. 402*

Modanlou, H. 404*, 405*

Mogerman, S. N. 367*

Moller, J. H. 300*

Molteni, A. 415*

Mondino, A. 60*

Monedjikova, V. 344*

Monkus, E. F. 308*, 314*, 381*, 396*, 405*, 769

Monset-Couchard, M. 638

Montalvo, J. M. 327*, 706

Monteleone, J. A. 330*

Montgomery, J. R. 365*

Moore, C. L. $386 *$

Moore, E. C. 362*, 367*, 369*

Moore, E. S. 409*, $411 *$

Moore, T. J. 418*, 422*

Moran, K. J. 374*

Morgan, S. K. 359*

Morin, C. L. $335^{*}$

Morishima, H. O. 299*, 397*

Morphis, L. G. 362*

Morriss, F. H., Jr. 314*, 794

Morriss, J. H. 306*

Morrissey, K. P. I19

Morse, C. W. 295*

Morse, P. A. 419*

Moses, S. W. 66*

Moshang, T., Jr. 330*

Mosovich, L. 334*, 601

Motoyama, E. K. $425 *$, 428*

Moxon, E. R. $377^{*}, 418^{*}$

Mucin 607
Mucolipidosis 560

Mucolipidosis III 751

Mucopolysaccharide 724

Mucopolysaccharidosis 560

Mudd, S. H. 390*, 645

Mukherjee, A. B. 696

Mukherjee, T. K. $320^{*}$

Müller, D. 53*

Müiller, H. 56*

Muller-Eberhard, U. 314*

Mumps 541

Munk, P. 331*

Murphcy, W. H. 64*, 65*, 601

Murphy, J. V. $347^{*}$

Murphy, M. G. 394*

Murphy, M. L. 352*, 361*, 388*

Murphy, S. 361*

Murray, K. 330*

Murthy, D. Y. N. 328*, 391*

Muscle, skcletal 787

Musgrave, J. E. 333*, 391*, 412*

Muzzo, S. J. 314*

Myocarditis 541

Nadas, A. S. $303 *$, 305*

Nadler, H. L. 346*, 419*

Naeye, R. L. $307 * 321 *, 405^{*}$

Nagel, J. E. 339*

Najarian, J. S. 292*

Najjar, V. A. $366^{*}$

Nakatake, T. 582

Nakatani, S. 346*, 392*

Nance, W. E. $346 *$

Nankervis, G. A. 376*

Naqvi, S. T. 413*

Nars, P. W. 63*

Nasopharynx 627

Nathan, D. 289*, 350*

Nathenson, G. 365*

Nave, E. 301*

Neal, W. A. $300 *$, 304*

Neel, J. V. 378*

Neerhout, R. C. $379 *$

Neims, A. H. $318^{*}, 320^{*}$

Nelson, N. 298*

Nelson, P. J. 296*

Nelson, T. 334*

Neonate 1, 20, 174, 769, 923, 937

Nephrotic syndrome 553

Nesbit, M. E. 349*

Neu, R. L. 344*

Neufeld, E. F. 57*, 342*, 343*

Neumann, C. G. 364*

New, M. I. 331*, 409*, 411*

Newberry, P. 297*

Newborn 82, 100, 184, 198, 527, 569, 575, $607,632,659,675,691,706,719,863$, $878,888,901$

Newman, A. J. 359*

Newton, C. $364 *$

Ng, W. G. 391*
Nicastri, A. 410*, 415*

Nichols, B. L. 331*, 340*, 384*

Nicolopoulos, D. 55*

Nicderwieser, A. 60*

Nielsen, S. 352*

Niemann, W. H. 397*

Nihill, M. R. $306^{*}$

Nir, E. 675

Niswander, J. D. 620

Nitrogen metabolism 161

Nitschke, R. 352*

Nixon, J. M. 406*

Nodari, S. 50*

Nocl, S. 302*

Nogrady, M. B. 408*

Nora, A. H. 321*

Nora, J. J. 304*, 306*, 321*

Norden, C. W. 377*

Nordio, S. 47*, 63*

Norepinephrine 575

Norman, M. 339*, 416*

Normand, I. C. S. 50*

Norris, D. G. 429*

Novak, M. 308*, 314*, 381*, $405^{*}, 769$

Nowak, J. 724

Nucleic acid synthesis 696

Nugent, E. W. 298*

Nyhan, W. L. 342*

O'Bell, J. C. $377^{*}$

Oberholzer, V. G. 728

O'Brien, J. 398*

O'Bricn, R. T. 343*, 358*, 359*

Ocampo, M. S. $409 *$

Ochs, H. D. $371^{*}$

Öckerman, P.-A. 52*

O'Connell, D. M. 430*

O'Connell, E. J. 424*, 429*

Ođell, G. B. $350 *$, $416 *$

Ogra, P. L. 292*, 377*

Oh, W. $311^{*}, 315^{*}, 325^{*}, 341^{*}, 396^{*}, 401^{*}$, $406 *, 870$

Oikawa, T. 401*

Okas, S. $365^{*}$

Okken, A. 52*, 863

Olafsson, A. 54*

Olambiwonnu, N. O. 331*

Olbing, H. 411*

Oldham, H. N., Jr. 305*

Olinsky, A. 291* , 430*

Oliver, W. J. 378*

Olley, P. M. $300^{*}, 425^{*}$

Olson, E. B., Jr. 395*

Olsson, T. 51*

Omori, K. 396*, 401*, 406* 870

O'Neal, P. 350*

Oppenheimer, E. H. 339*

Orange, R. 429*

Ordonez, J. V. 340*

O'Regan, S. 359*

O'Reilly, R. J. 369* 
Orcllana, T. 289*

Orenstein, D. M. 430*

Orgel, H. A. 368*

O'Riordan, A. C. 304*

Ortega, J. A. 352*, 361*

Orth, D. $407^{*}$

Orzalesi, M. $50 *, 54^{*}, 428 *$

Oski, B. F. 298*, 322*

Oski, F. A. 291*, 294*, 298*, 322*, 350*, $361 *, 401 *, 427 *$

Oslander, L. 89

Osteogenesis imperfecta 757

Ostrea, E. M., Jr. 350*, 355*

Osztovics, M. 56 *

Othersen, H. B. 359*

Otten, A. $405^{*}, 407 *$ * $431 *$

Ottenbreit, M. J. 349*

Ottesen, E. 372*

Outerbridge, E. W. $430 *$

Overall, J. C., Jr. 378*

Owen, G. M. 296*, 297*, 359*, 392*

Oxygen toxicity 607

Orand, P. T. 309*

Pachman, L. M. 364*

Paciuc, S. 220

Pagel, C. 354*

Pagliara, A. S. $381 *, 388 *, 500$

Pagtakhan, R. D. 20

Paisley, J. W. 318*

Pajno-Ferrara, F. $57^{*}, 198$

Palmarino, R. 54*

Palmer, C. G. 351 *

Palmer, J. 428*

Palmer, 'T. 728

Palmitic acid 192

Palumbo, P. J. 326*

Pan, M. 299*

Pancreas 198

Pancreozymin 198

Panganiban, C. 428 *

Papagcorgiou, A. 184, 382*, 393*

Papageorgiou, P. 366*, 369*

Parathyroid hormone 485, 878

Pardo, V. 417*

Parke, J. C., Jr. 103, 370*

Parker, C. W. 315*

Parker, M. L. 328*

Parkinson, D. K. 324*

Parkman, P. D. 289*, 370*, 378*

Parkman, R. 289*

Parks, G. A. 331 *

Parks, J. 339*

Parks, J. S. 330*

Parnley, R. T. 359*

Parrott, R. H. 373*

Pariin, J. C. $319 *, 339 *$

Partin, J. S. 339*

Pask, B. A. 382*

Patchen, L. I. 601
Patel, M. S. 832

Paton, J. B. 321*, 405*, 411*, 418*, 427*

Patten, B. M. 421*

Paul, B. 314*

Paul, M. H. 312*

Paulini, A. 66*

Paulsen, E. P. 325*

Paunier, L. 47*, 59*, 383*, 983

Pearse, R. G. 392*

Pearson, H. A. 343* 358*, 359*

Pcden, V. H. 330*

Penicillin antibodies 675

Penn, D. 308*, 314*, 381*, 405*

Penny, R. 331*

Peralta, H. J. 541

Percy, A. K. 812

Pereira, C. $395 *$

Perera, D. R. $335^{*}$

Pcrez, D. 360*

Perhecntupa, J. 59*, 66*

Periodic breathing 174, 569

Perkins, H. 369*

Perlstcin, P. H. $312 *, 405^{*}, 406^{*}$

Peroxisomes 798

Persky, V. W. 364*

Persson, B. $49^{*}, 58 *$

Pescuc, A. 553

Peter, G. 369*, 378*

Peter, J. B. 379*

Petersen, P. M. 345*

Peterson, J. A. 345*

Peterson, R. 409*

Petit, P. 57*

Petrelli, M. 383*

Petrovic, M. $331^{*}$

Petty, R. E. 363*

Phang, J. M. 316*

Phelps, D. L. 3I1*, 34I*, 396*, 40I*, 406*, $407^{*}$

Phenylalanine hydroxylase I

Phenylkctonuria 588

Phibbs, R. 403*

Philip, A. G. S. 406*

Philippart, M. 346* 348*, 389*, 392*

Phillips, P. E. 378*

Philpott, G. W. 315*

Phosphoenolpyruvatc-carboxykinase 75

Phosphorus 485

Picecc-Bucci, S. 50 *

Pick, T. E. 359 *

Pickering, R. J. 362*

Piel, C. F. 392*

Pierce, R. W. 691

Pinsky, L. 332*

Piomelli, S. 322*, 350*, 360*

Phosphorylcholine glyceride transferase 632

Pituitary $71,527,948$

Pituitary physiology 28

Placenta 139, 192, 509, 541, 706, 719, 798, $826,908,965$

Plato, C. C. 111, 620
Plunket, D. C. $374^{*}$

Poley, J. R. 335*, 340*

Pollack, J. D. 392*

Pollara, B. 362*, 367*

Pomerance, J. J. 406*, 422*

Pompe's disease 224

Porter, B. A. 332*

Porter, F. S. 954

Porter, I. H. 314*

Porter, P. N. 307*

Potter, D. E. 415*

Powars, D. $349^{*}, 351^{*}$

Powell, H. $407^{*}$

Pregnancy 509

Premature infant 198, 675

Prematurity 888

Prenatal diagnosis 958

Prensky, A. L. 418*

Presente, E. 393*

Price, R. A. $370^{*}$

Pricst, J. H. 382*

Prince, P. E. 421*

Prod'hom, L. S. 50*, 126, 888

Progesterone 948

Prolactin 527

Proline 818

Propranolol 931

Prostaglandin $\mathrm{E}_{1} 878$

Protein synthesis 923

Proteinuria 553

Pruitt, A. W. 321*

Puck, T. T. 344*

Punnett, H. H. 348*, 368*

Pupene, M. B. 994

Purugganan, G. 368*

Purvis-Smith, S. G. 215

Pyesmany, A. F. 371*

Pyruvate carboxylase 832

Pyruvate dehydrogenase 616

Pyruvate kinase 75

Qazi, Q. H. $375^{*}, 421^{*}$

Quan, T. 372*

Quic, P. G. 371*

Quinlivan, W. L. 331*

Rabenowiz, K. 383*

Rabin, P. 394*

Rachclefsky, G. S. 371*

Radde, I. C. $398^{*}$

Radin, N. S. 383*

Ragab, A. H. 351*

Rahiala, E.-L. 309*

Rahill, W. J. 314*, 415*

Räihä, N. C. R. 1, 58*, 309*, 316*, 527, 908

Rajegowda, B. K. 320*

Rallison, M. L. 321*

Ramos, C. V. 330*

Randolph, J. 429*

Rao, A. R. 353* 
Rao, L. M. $354 *$

Rao, P. S. $304 * 305^{*}$

Rappeport, J. 289*

Rappaport, R. 322*

Rausen, A. R. 412*

Rawnslcy, E. 348*, 394*

Ray, M. $347^{*}$

Razook, J. 305*

Rea, C. 818

Read, J. E. 422*

Read, S. E. 412*

Reber, R. 332*

Redding, R. A. 395*

Reddy, B. N. 362*

Reed, C. E. 343*

Recd, G. 302*

Recs, W. C. $365^{*}$

Refctoff, S. 332*

Regen, D. M. 418*, 422*

Reilly, E. L. 419*

Reimold, E. WV. $416^{*}$

Reinhart, J. A. 410*

Reisner, S. H. 184, 382*, 393*

Rciss, H. E. 55*

Reiter, E. O. 323*

Relier, J. P. 407*

Renal tubular aciclosis 832

Renaud, L. 414*

Rennert, O. M. 336*, 387*, 394*, 395*

Renton, K. W. $317^{*}$

Repletion 778

Resistance, airway 627

Resnick, J. S. 315*

Respiration 20, 174

Respiratory center 20

Respiratory distress syndrome $82,632,638$

Reyes, F. X. 306*

Reynolds, D. W. 372,* 379*

Reynolds, E. O. R. 50*

Reynolds, L. W. 751

Reynolds, W. A. 345*

Ribich, W. N. 403*

Ribosome 5

Rich, H. 295*

Richards, C. 326*, 328*

Richardson, C. J. 406*, 422*

Richardson, F. 422*

Rickets 914

Riegel, K. P. 51*

Rifkind, A. $315^{*}$

Rigatto, H. 430*

Riggio, R. R. $411^{*}$

Riley, F. C. 757

Rimoin, D. L. 348*

Ripley, B. A. 394*

Risi, J. 376*

Rittmanic, T. 372*

Ritz, E. 46*

Riva, E. 60*

Rivaxd, G. E. 352\%

Rivers, R. P.A. 50*
RNA 696

Robbins, J. B. 103, 370*

Roberts, N. K. 305*

Robertson, W. v. B. 346*

Robillard, J. E. 416*

Robinow, M. 348*

Robinson, A. 344*

Robinson, R. G. 340*

Roboz, J. 58*

Robson, A. M. 409*, 413*

Rodnan, J. B. 394*

Rodriquez, J. T. 340*

Rodriguez-Martinez, F. 627

Roe, C. R. 305*, 392*

Roehling, T. 174

Roerdink, F. H. 863

Roffwarg, H. P. 323*, 328*

Roghmann, K. J. 297*, 520

Roginsky, M. 393*, 394*

Roloff, D. W. 321*

Rommel, K. 62*

Romshe, C. A. $388^{*}$

Roncari, D. A. 417*

Roof, B. S. $392 *$

Root, A. W. 332*, 397*, 412*

Root, R. K. 352*

Rosalinas-Bailon, A. 322*

Rosan, R. C. $406^{*}$

Rosanda, C. 62*

Rose, A. 295*

Rose, A. L. $422 *$

Rose, V. 392*

Rosecrans, C. 351 *

Rosen, J. F. 393*

Rosen, M. R. 300*

Rosenberg, J. B. 293*

Rosenberg, L. E. 291*, 342*, 388*

Rosenblatt, R. M. 381*

Rosenbloom, A. L. 333*, 393*, 395*

Rosenfeld, C. R. 139

Rosenficld, R. L. 332*, 414*

Rosengart, R. 401*

Rosenow, E. C., III 424*, 429*

Rosenquist, G. C. 312*

Rosenthal, A. $301 * 303 *$

Rosenthal, I. M. 422*

Rosman, B. 294*

Rosner, A. 298*

Rosso, P. 314*

Roth, J. 291*

Roth, J. C. 290 *

Roth, M. M. 320*

Rothberg, R. M. 364*

Rothman, J. P. 412*

Rothner, A. D. 422*

Rowe, M. I. $411^{*}$

Rowe, R. D. 298*

Rowe, V. E. 322*

Rowland, L. P. 739

Rowland, T. W. 305*

Rowley, P. T. 351*
Roy, C. C. $335^{*}$

Rubella, congenital 215

Rubin, A. L. 411*

Rubin, H. M. $330 *$

Rudloff, B. 367*

Russell, P. S. 290*

Russell, R. 430*

Rutzky, J. 352*

Ryan, J. M. 378*

Saarikoski, S. 329*, 908

Sack, J. $325^{*}$

Sackett, D. L. 297*

Sadeghi-Nejad, A. 348*, 393*

Sacnger, P. $411^{*}$

Sagel, I. 416*

Sahn, D. J. 301*

Saini, I. 352*

Salmi, T. T. 57*

Salmon, S. 369*

Samaha, F. J. 423*

Samloff, I. M. $338^{*}$

Sandberg, D. H. 297*, 386*

Sandman, R. $346^{*}$

Santilli, J. 365*

Santos, A. Q. 312*

Sanyal, S. K. $305^{*}, 431^{*}$

Sarcione, E. 316*

Sarkar, D. B. 305*

Sarraf, S. $406^{*}$

Sarty, M. 421*

Sauberlich, H. E. 337*

Saunders, E. F. 356*

Savage, D. C. L. 291*

Savignoni, P. G. 50*

Sawaf, H. 352*

Sawitsky, A. 360*

Scanlon, R. T. $365^{*}$

Scarabino, R. 54*

Schacht, R. G. 416*

Schafcr, I. A. 313*, 383*, 965

Schaffer, D. 403*

Schaffner, F. 347*

Schalk, U. 174

Schärer, K. $46^{*}$

Schaub, J. 66*

Scheiner, A. P. 349*

Schiff, D. 184, 311*, 382*, 393*

Schleman, M. 303*

Schlote, W. $53^{*}$

Schmaier, A. H. 360*

Schmerler, A. $431^{*}$

Schmickcl, R. D. 5, 316*, 393*

Schmid-Rütcr, E. 6弓*

Schneerson, R. 103, 370*

Schneider, A. J. $380 *$, 394*

Schneider, G. 332*

Schncider, H. 192

Schncider, J. A. 291*, 395*

Schocn, E. J. 317*

Schocneman, M. 340* 
Schofield, B. H. 13

Schön, R. 64*

Schonberg, S. K. 334*

Schonberger, L. 392*

Schonland, M. 370*

Schorr, J. B. $360 *$

Schorr, P. 360*

Schroeder, W. 351*

Schröter, W. $61^{*}$

Schubert, W. K. 319*, 339*, 355*, 389*

Schüler, H. 46*

Schulkind, M. L. 369*

Schulte, F. J. $51 *, 588$

Schultz, W. F. $377^{*}$

Schussler, G. C. $325^{*}$

Schuster, S. R. 424*

Schuster, W. 45*

Schwartz, A. D. 358 *

Schwartz, E. 311*, 352*, 360*

Schwartz, E. 411*

Schwartz, R. 329*

Scofield, G. F. 364*

Scopes, J. W. 54*

Scott, C. R. $410^{*}$

Scott, R. B. $360^{*}$

Scott, T. F. M. $407 *$

Scriver, C. $382 *, 387 *, 390 *, 409 *$

Scriver, C. R. 149, 184

Scully, K. J. 388*

Sealy, W. C. $305 *$

Secretin 198

Sedaghatian, M. R. 398*, 431*

Sedaghatian, R. 400*

Seddon, J. M. 298*

Seclig, M. S. $378^{*}$

Segal, S. 818

Segel, G. B. 351 *

Seidel, H. M. 298*

Seifert, C. 330 *

Seip, M. F. 289*

Sekadde, C. B. 333*

Sela, M. 675

Seligman, F. 297*

Sell, E. J. 354*

Sen, L. 366*

Senior, B. 348*, 393*

Senior, J. M. $354 *, 427 *$

Sensenbrenner, J. A. 560

Sensirivatana, R. 417*

Serine 527

Serotonin 588

Sessoms, C. $336^{*}$

Settergren, G. 49*

Seungdamrong, S. 416*

Sevanian, A. 424*

Severi, F. 66*

Seyer, J. M. $306^{*}$

Shaffer, C. L. $354^{*}$

Shah, L. J. $352 *$

Shah, N. 360*

Shah, N. K. 353*
Shahidi, N. T. $354 *$

Shamsuddin, M. 351*

Shannon, D. C. $423 *$, 431*

Shapira, Y. 423*

Shapiro, B. H. 323*

Sharma, M. K. $368^{*}, 375^{*}$

Sharp, G. 300*

Sharp, H. L. 389*

Shaw, K. N. F. 59*

Shaywitz, B. A. 421*

Shearer, W. T. 315*

Sheepers, J. 398*

Shelley, H. J. 47*

Shende, A. 360*

Sherman, J. O. $389 *$

Shih, V. E. $382 *$

Shinefield, H. R. 367*

Shore, N. A. 353*, 361*

Shott, R. J. 379*

Shulman, S. T. 364*

Shumak, K. H. $367^{*}$

Shumway, C. N. $354 *$

Shurtleff, D. B. 410*

Shwachman, H. 293*, 335*, 337*

Sia, C. $402^{*}$

Siassi, B. 206*, 404*

Sibinga, M. S. 295*, 298*, 320*, 336*

Sick role behavior 520

Sidbury, J. B. 322*, 392*

Sidhu, J. $361^{*}$

Siegel, E. 119

Siegel, N. J. 290*, 402*, 417*

Siegel, S. E. 36 I $^{*}$

Siegel, S. R. 315*

Sieger, L. 361*

Sierralta, W. 326*

Siggers, D. $348^{*}$

Siimes, M. A. $315^{*}$

Silberberg, R. 348*

Silver, H. K. 298*

Silver, L. 353*

Silverberg, R. L. 290*

Silverman, J. A. 383*

Simberkoff, M. S. $373 *$

Simel, O. 59*

Simian line 111

Simmons, M. A. $309^{*}$

Simons, R. L. 292*

Sinaiko, A. R. 417 *

Singh, J. 384*

Singh, S. 318*

Sinks, L. F. 316*

Sirmokadam, B. R. 341*

Sisco, F. 370*

Sisson, T. R. C. $316^{*}, 397^{*}, 423^{*}$

Sizonenko, P. C. $47 *$, 59*, 322*, 383*, 983

Skala, J. P. 381*

Skeletal development 485

Sklenovsky, A. 20

Skowsky, R. 333*

Slaunwhite, W. R., Jr. 333*
Sleep 588

Sly, W. S. $342 *$, 384*

Smith, A. L. 318*, 377*, 380*, 418*

Smith, B. T. $308^{*}$

Smith, D. H. 318*, 369*, 377*, 378*, 380*, 413*, 418*

Smith, D. W. 423*

Smith, E. 299*

Smith, F. G., Jr. 333*, 416*

Smith, J. D. 340 *

Smith, P. B. 373*

Smithwick, M. 375*

Smitten, L. 729

Smythe, P. M. 370*

Snodgrass, P. J. 381*

Sobel, E. H. 331*

Solomon, I. L. 317*

Solomons, C. C. $361 *$

Sonneborn, M. 812

Soodalter, J. 569

Sosa, R. 396*

Soukup, S. M. 348*, 389*

South, M. A. 365*

Sowers, L. H. 945

Soyka, L. F. 317*, 321*, 322*

Spargo, B. H. 290*

Sparkes, R. S. $346 *$

Spector, E. B. $342 *, 700$

Spence, M. W. 394*

Spencer, M. L. 326*

Sper, M. R. 389*

Sperling, M. A. 311*, 407*

Sphingolipid hexose 812

Sphingolipidosis 560

Spicer, S. S. $359 *$

Spigland, I. 334*, 370*

Spirito, R. A. 307*

Spitler, L. E. 367*

Spitzer, A. 408*, 411*, 413*

Spotnitz, H. M. 300*

Springrose, S. 362*

Sprunt, K. 379*

Stagno, S. 372*, 379*

Stahl, M. 62*

Stahl, P. D. $384 *$

Stahlman, M. 405*, 407*, 426*, 431*

Stalder, G. R. 56*

Standaert, T. A. 302*

Stankewick, W. R. 301*

Starfield, B. H. 298*

Stave, U. 309*

Steeg, C. N. 299*

Steele, J. M., Jr. 416*

Steele, M. W. 344*, 394*

Steele, R. W. 372*, 379*

Steerman, R. 368*

Stegink, L. D. $386^{*}$

Steichen, J. J. 323*

Steinberg, F. S. 111

Steiner, P. 387* 
Steinfeld, L. 299*, 344*

Steinschneider, A. 293*

Stcisel, I. M. 293*

Stenzel, K. H. 411*

Stern, L. 184, 382*, 393*, 395*, 430*

Stern, R. C. $430^{*}$

Stcrnlieb, I. 386*

Sternowsky, H. J. 5̌**, 316*

Stevens, F. 296*

Stevenson, J. 309*

Stewart, C. $315^{*}$

St. Geme, J. W., Jr. 379*, 541

Sticklcr, G. B. 485, 978

Stiehm, E. R. 364*, 369*, 371*, 380*

Stinson, D. 826

Stites, D. P. 362*, 367*

St. Martin, E. C. $347^{*}$

Stockert, J. E. 300*, 301*

Stockman, J. A. 361*

Stoncbraker, F. E. 377*

Stoner, G. E. 35 †*

Stopa, A. 332*

Storage disease 13

Strauss, A. W. 306*, 431*

Strauss, J. 411*, 417*

Stxauss, R. G. 350*

Streeter, S. A. 342*

Stress 139,520

Strieder, D. 424*

Stringfellow, D. A. 371*, 378*

Strong, W. B. 305*

Strott, C. $407^{*}$

Stuart, M. J. 361*

Stubbe, P. 58*

Stubbs, K. G. 372*

Sturman, J. A. $58 *, 307 *, 347 *, 527,908$

Stutzman, L. 353*

Suh, S. M. 324*

Sujansky, E. 343*

Sullivan, D. B. $363^{*}$

Sullivan, J. S. 383*

Sulyok, E. 888

Sumncrs J. 404*, 407*

Sundell, H. 407*, 426*

Susin, M. 409*

Sutherland, J. M. 312*, 404*, 405*, 406*

Sutton, A. 47*

Sutton, E. L. 322*

Suzuki, M. $417 *$

Swarner, O. W., Jr. 407*

Sweat 888

Swect, A. X. 399*, 403*

Swender, P. T. 379*

Swenseid, M. 364*

Swyer, P. R. 430*

Sydney line 111

Sympathetic nervous system 931

Szczepanik, P. A. 340*, 341*

Szendro, P. 326*

Szygenda, S. A. 338*
Tabor, E. 379*

Tacusch, W., Jr. 425*

Talamo, R. C. $343^{*}$

Tallan, H. H. 386*

Talner, N. S. 357 *

Talwalkar, Y. B. 333*, 391*, 412*

Tamura, T. 582

Tan, C. $352 *, 361^{*}$

Tanaka, K. 382*

Tang, S. 327*, 384*

Tannenbaum, S. 366*

Tashjian, A. H., Jr. 326*

Tate, J. 296*

Taussig, L. M. 336* $340^{*}$

Taylor, A. 303*

Taylor, A. 340*

Taylor, H. A. 13, 751

Taylor, J. F. N. 301*

Taylor, P. M. 362*

Tay-Sachs clisease 812

Teberg, A. J. 397*, 424*

Tecilazich, D. $47 *$

Tedesco, T. A. 348*, 394*

Teller, W. 62*

Teller, W. M. 100

Tempio, C. 296*

Tenckhoff, H. 413*

Terán, C. $326^{*}$

Terasaki, P. I. $371^{*}$

ter Meulen, V. 53*

Testosterone 948

Thach, B. T. 419*

Thaler, M. M. 308*, 334*, 337*, 356*, 401*

Thalhammer, O. 66*

Thapax, M. K. $305^{*}$

Thermoregulation 888

Thiamine 616

Thibeault, D. W. 407*

Thilenius, O. G. 299*

Thiolase 149

Thiry, L. 57*

Thom, M. S. 432*

Thomas, F. 358*

Thomas, G. H. 751

Thomas, J. 316*, 336*, 358*, 385*, 394*

Thompson, J. B. 340*

Thompson, R. I. 349*

Thompson, W. M., Jr. 305*

Thong, Y. H. 379*

Thrupp, L. D. 318*

Thymus 778

Thyroid 870

Thyroid diseases 28

Thyroiditis 28

Thyrotropin 28

Thyroxine 706

Tibbles, J. A. R. 349*

Ticpolo, L. 66*

$N$-Tiglylglycine 149

Tildon, J. T. 309*

Tillmann, W. 61*
Ting, P. 398*

Ting, R. Y. 298*

Tiwary, C. M. 333*, 393*, 394*, 395*

Tocci, P. M. $386^{*}$

Todd, J. K. 379*

Todd, T. 294*

Todres, I. D. 423*, 431*

Tomasi, T. B., Jr. 292*

Tonda, G. P. 62*

Torday, J. S. 308*

Tori, C. A. 82,569

Toskes, P. P. 336*

Townes, P. L. 349*

Traber, D. L. $410 *$

Trachea 607

Trihexosylceramide 684

Trainor, E. J. 429*

Triiodothyronine 706

Trisomy 5

Trouy, R. L. $325^{*}$

Trucconc, N. J. 300*, 931

Tsang, R. C. $323^{*}, 383^{*}, 387 *, 403 *$, 408*

Tsiantos, A. 407*, 426*

Tucker, A. S. 430*

Tung, K. S. K. 330*

Ty, A. 417*

Tyrosine transaminase 75

Uhlendorf, B. W. 645

Umana, G. 377*

Underwood, L. E. 324*

Uxine 724

Urmson, J. 350*

Usher, R. H. 399*, 400*, 408*

Uterine blood flow 139

Utcrus 870

Vagelos, P. R. 41 \%*

Vagina 139

Vagnucci, A. 329*

Vaidya, V. 330*

Vainsel, M. 47*

Valdes-Dapena, M. 304*

Valle, D. L. 316*

Valloton, M. B. 59*, 383*, 983

Vamos-Hurwitz, E. 57*

Van den Berghe, G. 59*

van der Blij, J. F. 52*, 863

Van Dyke, D. H. 424*

Van Dyck, O. B. 407*

Vannucci, R. C. 424*

Van Wyk, J. J. 324*

Varghese, P. J. 298*

Vasopressin 878

Vassella, F. 52*

Vats, T. S. 340*

Vaughan, M. 391*

Vcale, A. M. O. $65^{*}$

Vegnente, A. 95

Verder, H. 57*

Verity, M. A. $423 *$ 
Vernier, R. L. 292*, 315*

Verroust, F. M. 291* 395*

Versmold, H. 51*

Vest, M. 54*

Vethamany, S. K. $349 *$

Vetrella, M. 95

Victorin, L. 405*, 407*, 426*, 431*

Vida, L. N. $351^{*}, 356^{*}$

Vincent, M. M. 372*, 379*

Vinson, W. C. $306^{*}$

Viral embryopathy 215

Virnig, N. L. 292*

Visakorpi, J. K. $59^{*}$

Vitale, L. F. 322*

Vitamin D 914

Voina, S. J. 324*

Volpe, J. J. 417*

von Berg, W. 527

Vouga, A. 47 *

\section{Wagner, V. 659}

Wagner-Barnack, M. 901

Wahner, H. W. 706

Waked, N. 60\%

Waldenström, J. 342*

Waldman, R. H. 372*

Waldo, A. L. 299*

Walker, W. A. $334^{*}$

Wallace, R. B. 377*

Wallace, W. 368*

Wallach, S. $385^{*}$

Walling, L. 389*

Walters, T. R. 359*, 362*

Wang, J. J. 316*

Wangensteen, O. D. 428*

Wannamaker, L. W. 372*, 375*

Wapnir, R. A. 341*

Wara, D. 369*

Ward, R. 380*

Warner, J. R. 325*

Warner, M. 318*

Warren, R. J. $343^{*}$

Warshaw, J. B. 308*, 324*

Wasserman, E. 294*, 408*, 416*

Waterson, J. R. 316*

Watkins, J. B. 341*

Watson, S. C. $304^{*}$

Wcbb, L. S. 364*

Webb, T. E. 294*

Wehrle, P. F. 318*

Weiffenbach, J. M. 419*

Weinberger, H. L. 298*

Weiner, L. 298*, 322*

Wcintraub, B. 327*

Weishuhn, E. J. 304*, 306*

Weiss, L. $345^{*}$

Weissberg, E. 380*

Weitzman, E. D. 420*

Wcitzman, M. $380 *$
Welch, J. P. 349*

Weldon, C. S. $306^{*}$

Weldon, V. V. $333 *$

Weller, G. M. 293*

Wells, C. R. 304*

Wellwood, H. 349*

Wen, C.-P. $34 I^{*}$

Wende, R. D. 289*

Wendenburg, A. 174

Wennberg, R. P. 334*, 397*

Werner, J. 48*

Wcrtelecki, W. 132, 620

Westberg, M. J. 354*, 429*

Whang, E. 410*

Wharton, B. 54*

Whceler, F. B. 38?*

Whisnant, J. K. 103

White, J. B. 318*

White, W. L. 406*

Whitman, V. 302*

Whitten, C. F. 313*

Wichser, J. 423*

Wick, H. 58*, 64*

Wickler, M. 316*

Wiedemann, E. $411^{*}$

Wiesmann, U. 52*

Wile, M. 296*

Wilfert, C. M. 364*, 380*

Wilkinson, G. R. 318*

Wilkinson, R. 349*

Williams, D. $375^{*}$

Williams, D. L. 327*

Williams, F. 54*

Williams, K. O. 361*

Williams, M. L. 379*

Williams, P. R. 341*

Williams, T. E. 352*

Willis, M. D. 426

Wilson, C. B. 352*

Wilson, J. T. 318*

Wilson, R. S. $324 *, 719$

Windthorst, H. 51*

Winegrad, A. I. 385*

Wingerd, J. 317*

Wingfield, B. S. $324 *$, 420*

Winslow, P. R. 336*

Winsor, E. J. 349*

Winter, J. S. D. $306^{*}, 948$

Winter, W. P. 316*

Wirth, F. H., Jr. 341*

Wiscman, D. H. 432*

Wisniewski, H. K. 422*

Witte, J. J. 289*

Wittig, H. J. 372*

Wittner, M. K. 372*

Wohl, M. E. B. 424*

Wöhler, J. 46*

Wohltmann, H. J. 388*

Wolcott, G. J. 729
Wolf, H. 58*, 769

Wolf, J. L. 307*

Wolf, R. O. $340 *$

Wolfe, H. 296*

Wolff, J. A. 360*

Wolff, P. H. 293*

Wolfsclorf, J. 432*

Wolk, T. 397*

Wong, K. Y. $355^{*}$

Wong, W. C. $409^{*}$

Wood, O. L. 394*

Wood, R. E. 427*

Woodhcad, A. P. 343*

Woodruff, C. W. 338*

Woody, N. C. 994

Woznicki, W. 355*

Wright, A. 380*

Wright, W. C., J1. 380*

Wu, B. $425 *$

Wu, P. Y. K. $397 *, 421 *, 424 *$

Wurzcl, H. 348*

Wyllie, R. G. 560

Wyszogrodski, I. 425*

Yaddanapudi, R. 341*

Yaffe, S. J. 308*, 317*, 319*, 320*, 334*

Yaish, H. 352*

Yamauchi, T. 381*, 541

Yamaguchi, K. 329*

Yanagi, R. 322*

Yao, A. C. $306 *$

Yeager, A. S. 381*

Yeh, M. 299*

Yeh, S.-Y. 405*

Yen-Watson, B. 403*

Yeung, C.-Y. $317 *$

Yoon, J. J. 408*

Young, G. W. 305*

Young, M. K. 787

Young, P. 350*

Younoszai, M. K. 342*

Yu, P. 346*

Yuceoglu, A. M. 408*, 416*, 417*

Zabriskie, J. B. 290*, 412*

Zachman, R. D. $395^{*}, 402 *, 432 *, 632$

Zahnd, G. 983

Zamet, P. 638

Zapol, W. 300*

Zarkowsky, H. S. 362*

Zeilstra, K. 348*, 392*

Zclson, C. 289*

Zepp, H. D. 370*

Zetterström, R. 60*, 342*

Zigler, J. S. 322*

Zinkham, W. H. 355*

Zoppi, G. 57*, 198

Zuelzer, W. W. 341*, 349*, 353*

Zwerdling, R. G. 424* 